\title{
Stability Analysis of Fisher Equation Using Numerical Galerkin Techniques
}

Saad A. Manna

Ahmed F. Qassem

College of Computer Science and Mathematics

University of Mosul, Iraq

\section{Received on: $13 / 07 / 2005$}

\section{Accepted on: 10/10/2005}

\section{Abstract}

We studied the stability of the steady state solutions for Fisher Equation in two cases, the First one with constant amplitude and we show that the steady state solution $u_{1}=1$ is always stable under any condition, but the other two solutions $u_{1}=0$ and $u_{1}(x)=A \cos (n \pi X)$ are conditionally stable.

In the Second case, we studied the steady state solutions for various amplitude by using two Methods. The First is analytically by direct Method and the second is numerical method using Galerkin technique which shows the same results, that is the steady state solution $u_{1}=1$ is always stable under any conditions, but the other two solutions $u_{1}=0$ and $u_{1}(x)=A \cos (n \pi X)$ are conditionally stable.

Keywords: Stability Analysis, Fisher Equation, Galerkin Technique.

$$
\begin{aligned}
& \text { تحليل الاستقرارية لمعادلة Gisher باستخدام تقنيات Galerkin العددية } \\
& \text { أحمد فاروق قاسم } \\
& \text { سعد عبد الله مناع } \\
& \text { كلية علوم الحاسبات واليراضيات، جامعة الموصل } \\
& \text { تاريخ قبول البحث: 2005/10/10 } \\
& \text { تاريخ استلام البحث:2005/07/13 } \\
& \text { الملخص }
\end{aligned}
$$

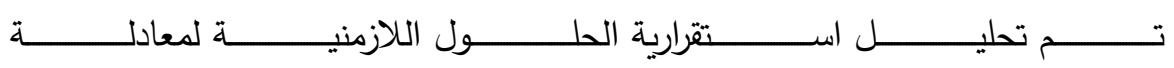

$$
\begin{aligned}
& u_{1}=1 \text { Fisher }
\end{aligned}
$$

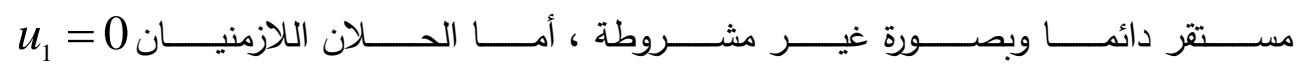

$$
\begin{aligned}
& \text { و (n) } \\
& \text { أما الحالة الثانية فقد تم دراسـة استقرارية الحلول اللازمنية في حالة كون السعة متغيرة } \\
& \text { وباستخدام طريقتين ، الأولى تحليلية ، والثانية طريقة عددية هي طريقة Galerkin إذ تم التوصل } \\
& \text { إلـى نفس النتائج ، وهـي أن الحسل اللازمنـي } \\
& \text { و ( } u_{1}(X)=A \cos (n \pi X) \text { فهما مستقران بصورة مشروطة. }
\end{aligned}
$$

الكلمات المفتاحية: تحليل الاستقرارية، معادلة Fisher، تقنية Galerkin. 


\section{Introduction}

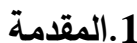

إن أي نظام (System) فيزيائي أو بايولوجي أو غيره إذا ما تعرض إلى تأثيرات خارجية

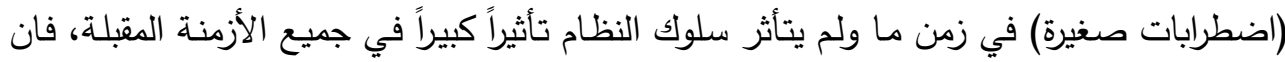
هذا النظام في حالة مستقرة (Stable)، فعلى سبيل المثال إن النظام الثمسي (Solar System) الموجود حاليا هو في حالة الاعتماد على الزمن الذي تتحرك فيه الكواكب حول الشمس وبشكل

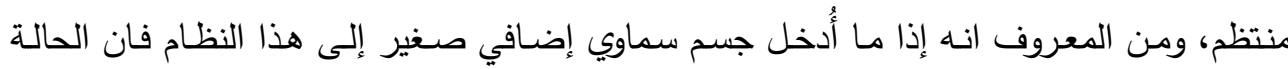

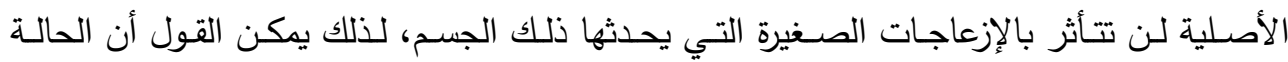
الأصلية مستقرة (Stable)، ويمكن أن تثار أسئلة مماثلة حول الاستقرارية في كل مشكلة فيزيائية أو

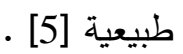

إن معادلة Fisher وضـعها العـالم فيشر في عـام (1937) إذ اقترحها بوصفها نموذجاً رياضياً لدراسة علم حركة السكان و انتشارهم [2].

في عام (2000)درس Gourley [4] حلول الموجة المنتقلة لمعادلة Fisher غير المحلية وهي حالة خاصة من معادلات الانتثار - التقاعل إذ لاحظ حلول الموجة المنتقلة التي تربط ما بين

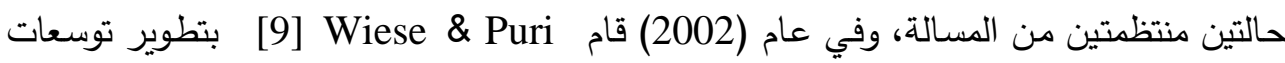

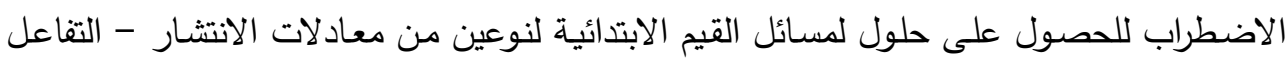
Ginzburg -Landau Fisher الأولى معادلة (Diffusion Equations) المعتمدة على الزمن،وفي عام (2004) درس Fort \& Mendez \& Ortega [7 دور زمن التأخير في نمذجة مدى التوسع البايولوجي لمعادلة Fisher ، لقد اثبت البحث بان زمندمن التبأخير

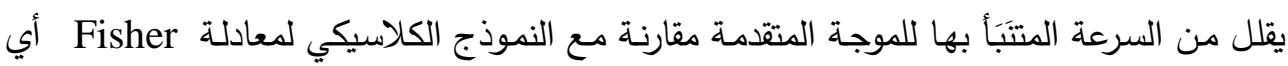
(معادلة Fisher من دون زمن التأخير ).

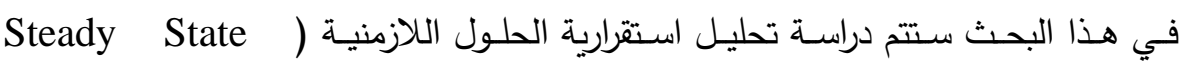
(Solutions السعة ثابتة، والثانية تتتاول دراسة تحليل الاستقرارية في حالة كون السعة متغيرة مع استخدام طريقة Galerkin

\section{Mathematical Model}

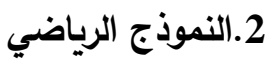

إن من معادلات الانتشار غير الخطية معادلة Fisher والتي صيغتها: 
$\frac{\partial u(x, t)}{\partial t}=D \frac{\partial^{2} u(x, t)}{\partial x^{2}}+h(u)$ حيث (u) =ru(1-u) هي دالة غير خطية تحقق الشروط الأتية:

$h(u) \in C^{1}([0,1])$

$h(0)=h(1)=0$

$h^{\prime}(0)=r>0$

$h^{\prime}(u)<r$

$u \in[0,1]$

إن u(x,t تمثل كثافة السكان (Population Density) عند الزمن (t) وعند الموقع

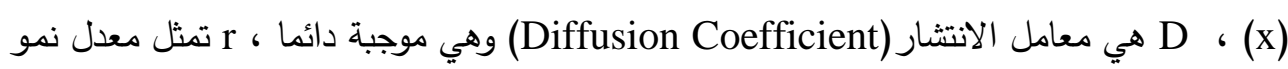
السكان (Growth Rate) وهي موجبة أيضا وتزداد إذا وجدت زيادة هائلة في السكان ، وهي تبين

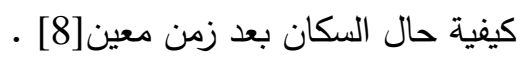

أمسا الثروط الحدودية والابتدائية المستخدمة في حل معادلة Fisher في النترة [0, x فهي الثروط الحدودية لنيومان (Neumann Boundary Conditions) وهي [3]: $\left.\begin{array}{l}\frac{\partial u(0, t)}{\partial x}=0 \\ \frac{\partial u(1, t)}{\partial x}=0\end{array}\right\}$

$u(x, 0)=f(x) \quad, x \in[0,1]$

لغرض إيجاد معادلة Fisher اللابعدية سوف نعرف القيم اللابعدية آلاتية[10, 11]:

$X=\frac{x}{L} \quad, \quad T=\frac{D}{L^{2}} t$

حيث L ت تمثل طول الفترة لـ أي أن:

$0 \leq x \leq L$

وباستخدام هذه التحولات نحصل على: 
$\frac{D}{L^{2}} \frac{\partial u}{\partial T}=\frac{D}{L^{2}} \frac{\partial^{2} u}{\partial X^{2}}+r u-r u^{2}$

$\Rightarrow \frac{\partial u}{\partial T}=\frac{\partial^{2} u}{\partial X^{2}}+\frac{r L^{2}}{D} u-\frac{r L^{2}}{D} u^{2}$

$\frac{\partial u(0, T)}{\partial X}=0 \quad, \quad \frac{\partial u(1, T)}{\partial X}=0$

المعادلة (3) مع الشروط الحدودية (4) تمثل الصيغة اللابعدية لمعادلة Fisher.

\section{Stability Analysis}

3. تحليل الاستقرارية

نفرض أن النظام قد تعرض إلى إزعاج، ونغرض أن

$u(X, T)=u_{1}(X)+u_{2}(X, T)$ و $u_{2}$ تمثل حالة الإزعاج المفروضة [5 ]:

$$
\begin{aligned}
& \frac{\partial u_{2}}{\partial T}=\left(\frac{d^{2} u_{1}}{d X^{2}}+\frac{\partial^{2} u_{2}}{\partial X^{2}}\right)+\frac{r L^{2}}{D}\left(u_{1}+u_{2}\right)-\frac{r L^{2}}{D}\left(u_{1}+u_{2}\right)^{2} \text { (3) نحصويض (5) في المعادلة (5) } \\
& \Rightarrow \frac{\partial u_{2}}{\partial T}=\frac{d^{2} u_{1}}{d X^{2}}+\frac{\partial^{2} u_{2}}{\partial X^{2}}+\frac{r L^{2}}{D} u_{1}+\frac{r L^{2}}{D} u_{2}-\frac{r L^{2}}{D} u_{1}^{2}-\frac{2 r L^{2}}{D} u_{1} u_{2}-\frac{r L^{2}}{D} u_{2}^{2}
\end{aligned}
$$

ويمكن فصل الحالة اللازمنية لمعادلة Fisher عن الحالة الزمنية وكما يأتي:

$0=\frac{d^{2} u_{1}}{d X^{2}}+\frac{r L^{2}}{D} u_{1}-\frac{r L^{2}}{D} u_{1}^{2}$

$\left.\frac{d u_{1}}{d X}=0 a t \quad X=0,1\right\}$

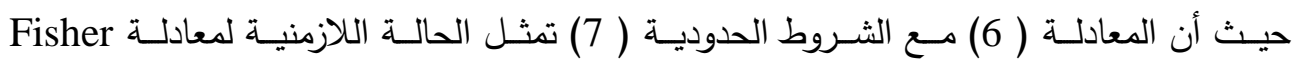

.(Steady State Of Fisher Equation)

$$
\begin{aligned}
& \frac{\partial u_{2}}{\partial T}=\frac{\partial^{2} u_{2}}{\partial X^{2}}+\frac{r L^{2}}{D} u_{2}-\frac{2 r L^{2}}{D} u_{1} u_{2}-\frac{r L^{2}}{D} u_{2}^{2} \\
& \left.\frac{\partial u_{2}(X, T)}{\partial X}=0 \quad \text { at } \quad X=0,1\right\}
\end{aligned}
$$


حيث أن المعادلة ( 8) مع الثروط الحدودية ( 9) تمثل الحالة الزمنية لمعادلة Fisher .

\section{Uniform Steady State Solution}

4.الحل الللازمني المنتظم:

المعادلة (6) مسع الثروط الحدودية ( 7) لها حلان

الثابتة (Constant Solutions) إذ أن

وللحصول على الحل غير الثابت Nonconstant Solution) للمعادلة (6) مع الثروط

الحدودية نلجأ إلى Linearized Stability Analysis للمعادلة (6) [5,10] لتصبح :

$\frac{d^{2} u_{1}}{d X^{2}}+\frac{r L^{2}}{D} u_{1}=0$

إن المعادلة ( 10) هي معادلة تفاضلية اعتيادية خطية ومتجانسة والحل العام لهذه المعادلة هو : $u_{1}(X)=A \cos M X+B \sin M X$

حيث أن

بتعويض الثروط الحدودية (7) في المعادلة (11)نحصل على:

$B M=0$

$-A M \sin (M)+B M \cos (M)=0$

$$
\begin{aligned}
& \text { من المعادلة (12) بما أن } 0=0 \text { إذن } \\
& \text { بالتعويض عن قيمة B في المعادلة ( 13) نجد أن : }
\end{aligned}
$$

$-A M \sin (M)=0$

$$
\begin{aligned}
& \text { حيث أن } 0 \text { حان } \\
& \text { إما } u_{1}=0 \text { =0 وهذا هو الحل التافه }
\end{aligned}
$$

$\sin (M)=0$

$\Rightarrow M=n \pi \quad, \quad n= \pm 1, \pm 2, \pm 3, \ldots$

$\Rightarrow \sqrt{\frac{r L^{2}}{D}}=n \pi \quad, \quad n= \pm 1, \pm 2, \pm 3, \ldots$ 
$\Rightarrow \frac{r L^{2}}{D}=(n \pi)^{2} \quad, \quad n= \pm 1, \pm 2, \pm 3, \ldots$

إذاً الحل اللازمني لمعادلة Fisher الذي يحقق الثروط الحدودية (7) هو: $u_{1}(X)=A \cos (n \pi X) \quad, \quad n= \pm 1, \pm 2, \pm 3, \ldots$

5. تحليل الاستقرارية في حالة السعة الثابتة(حالة الاضطراب):

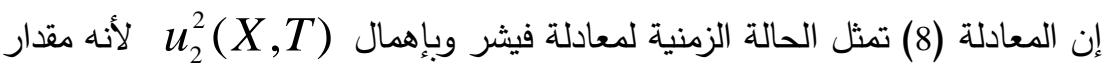

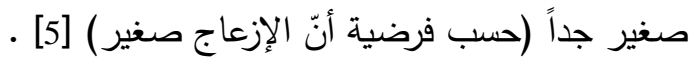

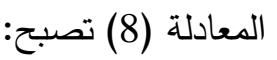

$\frac{\partial u_{2}}{\partial T}=\frac{\partial^{2} u_{2}}{\partial X^{2}}+\frac{r L^{2}}{D} u_{2}-\frac{2 r L^{2}}{D} u_{1} u_{2}$

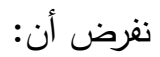

$u_{2}(X, T)=\mathrm{P} e^{i k(x-c t)}$

حيث أن

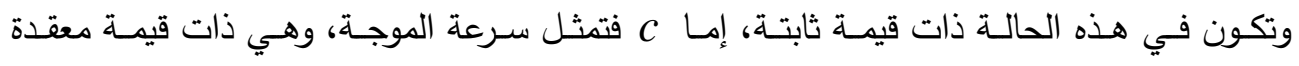
(Complex) تؤدي إلى نمو الإزعاج أو تلاشيه على التوالي ، فعندما تكون

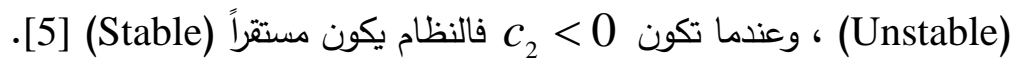
بتعويض الفرضية ( 16) في المعادلة ( 15) نجد أن : $-i k c P e^{i k(x-c t)}=i^{2} k^{2} P e^{i k(x-c t)}+\frac{r L^{2}}{D} P e^{i k(x-c t)}-\frac{2 r L^{2}}{D} P e^{i k(x-c t)} u_{1}(X)$

يمكن تبسيط المعادلة السابقة لتصبح:

$-i k c=-k^{2}+\frac{r L^{2}}{D}-\frac{2 r L^{2}}{D} u_{1}(X)$

$$
-i k\left(c_{1}+i c_{2}\right)=-k^{2}+\frac{r L^{2}}{D}-\frac{2 r L^{2}}{D} u_{1}(X)
$$

$\Rightarrow-i k c_{1}+k c_{2}=-k^{2}+\frac{r L^{2}}{D}-\frac{2 r L^{2}}{D} u_{1}(X)$ بعد فصل الجزء الحقيقي والجزء الخيالي نحصل على:

$-i k c_{1}=0$ 
$k c_{2}=-k^{2}+\frac{r L^{2}}{D}-\frac{2 r L^{2}}{D} u_{1}(X)$
$\Rightarrow c_{2}=\frac{-k^{2} D+r L^{2}-2 r L^{2} u_{1}(X)}{k D}$

بما أن c هي عامل التضخم Amplification Factor) التي تؤدي إلى تحديد حالة

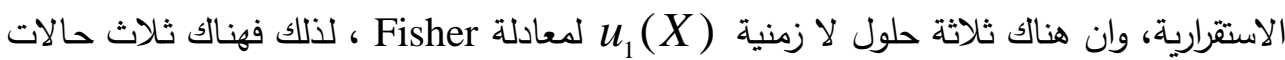

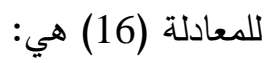

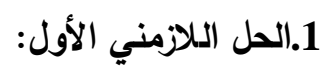
عندما تكون $c_{2}=\frac{-k^{2} D+r L^{2}}{k D}$ وبذلك يكون الحل $k^{2} D>r L^{2}$ $L^{2}>0, D>0, r>0$ حيث

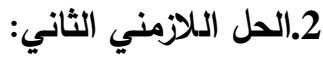
عندما

$c_{2}=\frac{-k^{2} D-r L^{2}}{k D}$

$\Rightarrow c_{2}=\frac{-\left[k^{2} D+r L^{2}\right]}{k D}$

بما أن هذه الحالة مستقراً دائما.

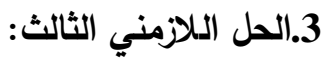
عندما يكون $c_{2}=\frac{-k^{2} D+r L^{2}-2 r L^{2} A \cos (n \pi X)}{k D}$

و ولإيجاد منحني الاستقرارية المتعادل (Neutral Stability Curve) نضع ونأخذ اقل قيمة ذاتية عندما يكون $n=1$ فنحصل من المعادلة (20) على: $k^{2} D=r L^{2}-2 r L^{2} A \cos (\pi X)$ 
$\Rightarrow k=\sqrt{\frac{r L^{2}-2 r L^{2} A \cos (\pi X)}{D}}$

حيـــث أن

$$
0 \leq X \leq 1 \quad \text { : }
$$

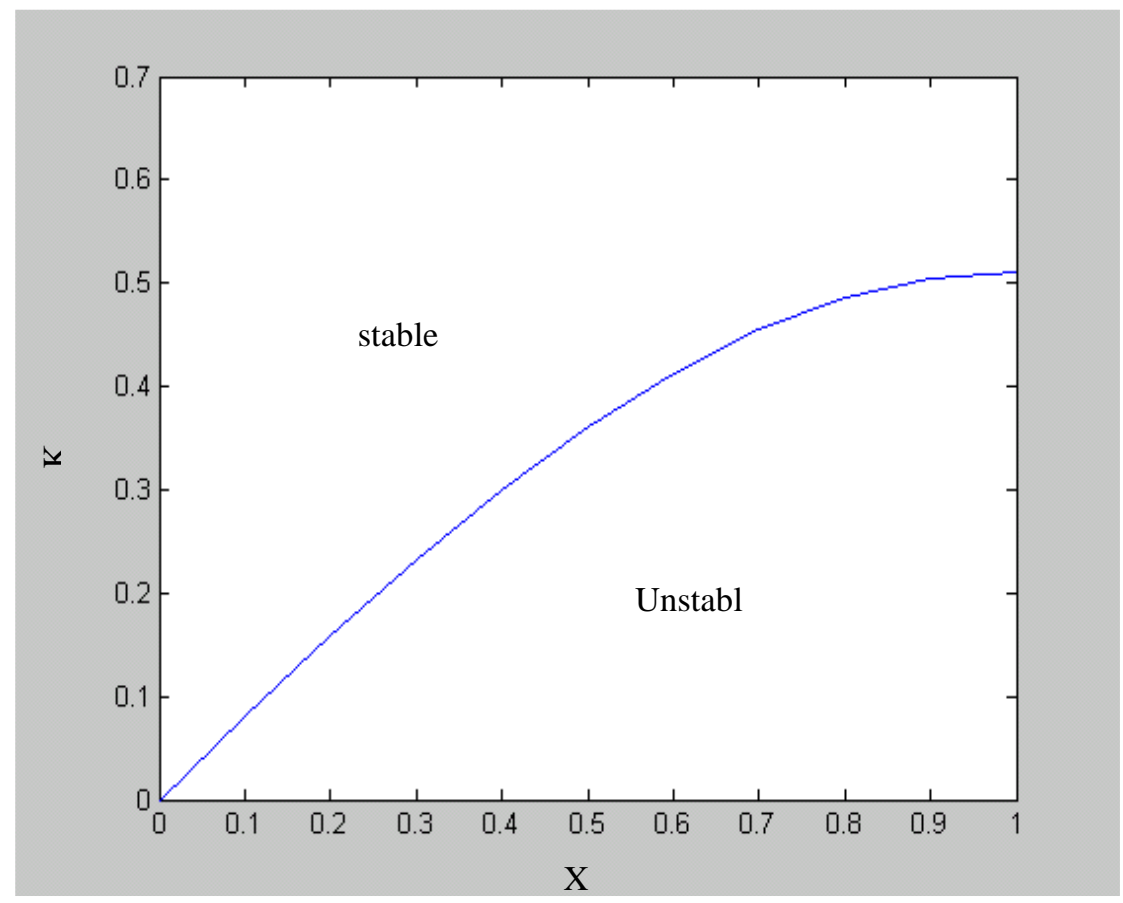

الثكل (1)

يمثل منحني الاستقرارية المتعادل للمعادلة (21)

عندما $A=0.5, L^{2}=1, r=1, D=1$,

6.تحليل الاستقرارية في حالة السعة المتغيرة(حالة الاضطراب):

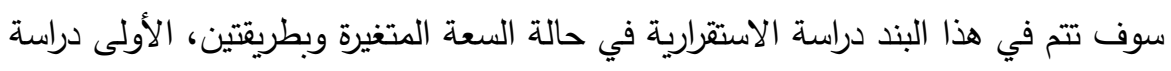

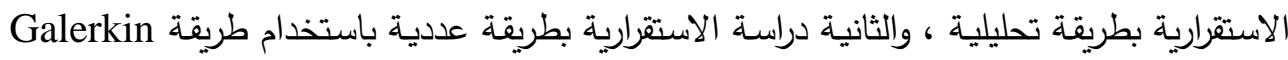
العددية .

نفرض أن

$u_{2}(X, T)=F(X) e^{i k(x-c t)}$ 
حيث أن

(Amplitude)

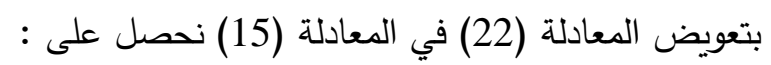

$-i k c F(X) e^{i k(x-c t)}=-k^{2} F(X) e^{i k(x-c t)}+i 2 k F^{\prime}(X) e^{i k(x-c t)}+F^{\prime \prime}(X) e^{i k(x-c t)}+$ $+\frac{r L^{2}}{D} F(X) e^{i k(x-c t)}-\frac{2 r L^{2}}{D} u_{1}(X) F(X) e^{i k(x-c t)}$ بعد تبسيط المقدار نحصل على:

$-i k c F(X)=-k^{2} F(X)+i 2 k F^{\prime}(X)+F^{\prime \prime}(X)+\frac{r L^{2}}{D} F(X)-$

$$
-\frac{2 r L^{2}}{D} u_{1}(X) F(X)
$$

$\Rightarrow-i k c_{1} F(X)+k c_{2} F(X)=-k^{2} F(X)+i 2 k F^{\prime}(X)+F^{\prime \prime}(X)+\frac{r L^{2}}{D} F(X)-$

$$
-\frac{2 r L^{2}}{D} u_{1}(X) F(X)
$$

$-i k c_{1} F(X)=i 2 k F^{\prime}(X)$ بعد فصل الجزء الحقيقي والجزء الخيالي نحصل على:

$k c_{2} F(X)=-k^{2} F(X)+F^{\prime \prime}(X)+\frac{r L^{2}}{D} F(X)-\frac{2 r L^{2}}{D} u_{1}(X) F(X)$

$\Rightarrow F^{\prime \prime}(X)+\left(\frac{r L^{2}}{D}-k^{2}-k c_{2}-\frac{2 r L^{2}}{D} u_{1}(X)\right) F(X)=0$

$F^{\prime}(X)=0 \quad$ at $X=0$ and $X=11^{\text {والثروط الحدودية (9) تصبح)...(25) }}$

\section{Analytical Solution}

أولا: الحل التحليلي

يمكن كتابة المعادلة (24) بالثكل الأتي :

$$
\begin{aligned}
& F^{\prime \prime}(X)+\lambda F(X)=0 \\
& \lambda=\frac{r L^{2}}{D}-k^{2}-k c_{2}-\frac{2 r L^{2}}{D} u_{1}(X)
\end{aligned}
$$

إن الحل العام للمعادلة (6 2) يكون بالصيغة آلاتية:

$F(X)=a \cos \sqrt{\lambda} X+b \sin \sqrt{\lambda} X$ 
بتعويض الشروط الحدودية (5 2) يمكن أن نحصل على

$$
\begin{aligned}
& b \sqrt{\lambda}=0 \\
& -a \sqrt{\lambda} \sin \sqrt{\lambda}+b \sqrt{\lambda} \cos \sqrt{\lambda}=0
\end{aligned}
$$

من المعادلة (29) ، بما أن إذاً المعادلة (30) تصبح: (29) ، بها ان

$-a \sqrt{\lambda} \sin \sqrt{\lambda}=0$

$$
\lambda>0
$$

$$
\sin \sqrt{\lambda}=0
$$$$
\Rightarrow \lambda=(n \pi)^{2}
$$

أو

أو إماث $n= \pm 1, \pm 2, \pm 3, \ldots$

من المعادلة (27) نحصل على :

$\frac{r L^{2}}{D}-k^{2}-k c_{2}-\frac{2 r L^{2}}{D} u_{1}(X)=(n \pi)^{2}$

$\Rightarrow c_{2}=\frac{-k^{2} D-2 r L^{2} u_{1}(X)-(n \pi)^{2} D+r L^{2}}{k D}$

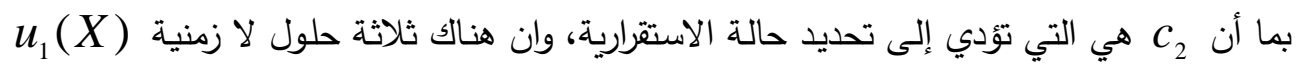
لمعادلة Fisher ، لذلك فإن هناك ثلاث حالات للمعادلة (31) هي: هي:

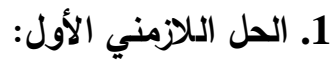
عندما $c_{2}=\frac{-k^{2} D-(n \pi)^{2} D+r L^{2}}{k D}$

وبنلك يكون الحل

$k^{2} D+(n \pi)^{2} D>r L^{2}$

$L^{2}>0, D>0, r>0$ 


$$
\begin{aligned}
& c_{2}=\frac{-k^{2} D-r L^{2}-(n \pi)^{2} D}{k D} \\
& \Rightarrow c_{2}=\frac{-\left[k^{2} D+r L^{2}+(n \pi)^{2} D\right]}{k D}
\end{aligned}
$$

بما أن بأ $u_{1}=1$

3. - I الحل اللازمني الثالث: عندما $c_{2}=\frac{-k^{2} D-2 r L^{2} A \cos (n \pi X)-(n \pi)^{2} D+r L^{2}}{k D}$

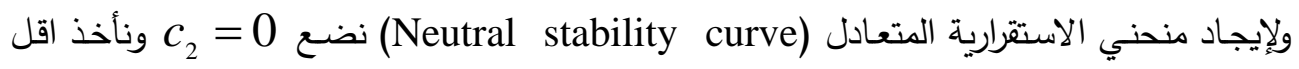

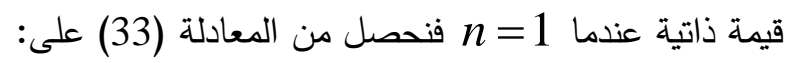
$k^{2} D=-2 r L^{2} A \cos (\pi X)-(\pi)^{2} D+r L^{2}$ $\Rightarrow k=\sqrt{\frac{r L^{2}}{D}-\frac{2 r L^{2} A \cos (\pi X)}{D}-9.8696}$

حيث أن

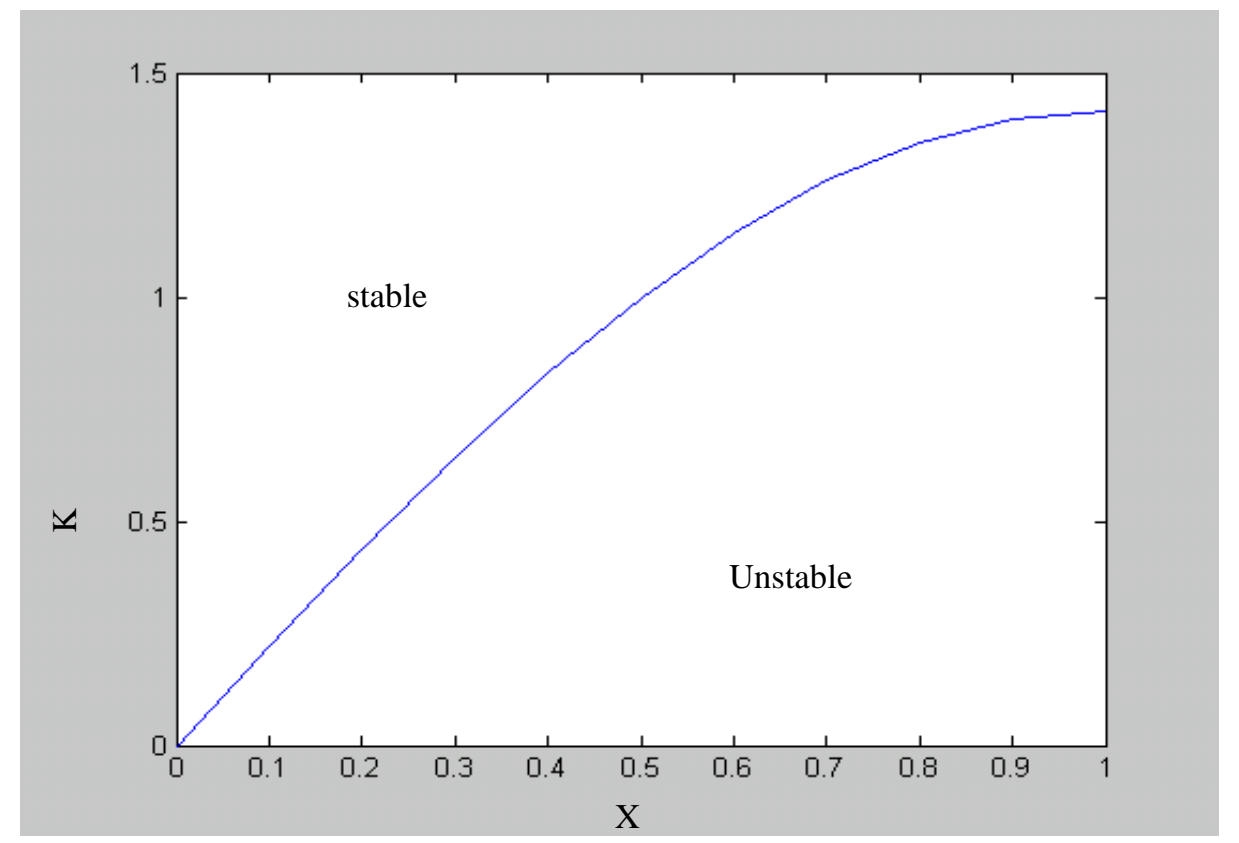

(2) الثكل

يمثل منحني الاستقرارية الوقوعادل للمعادلة (34)

$A=0.00652, L^{2}=1, r=10, D=1$ عندمان 
ثانيا: تقنية Galerkin العددية

سوف يتم إيجاد حل عددي للمعادلة (24) مـع الثـروط الحديـة (25) باستخدام طريقة

Galerkin

نفرض أن الحل للمعادلة (26) بالثكل الأتي :

$F(X)=\sum_{v=1}^{\infty}\left(A_{v} \cos \lambda_{v} X+B_{v} \sin \lambda_{v} X\right)$

بأخذ أحد حدود الحل السابق للمعادلة (35) نحصل على :

$F(X)=A_{v} \cos \lambda_{v} X+B_{v} \sin \lambda_{v} X$

وباستخدام الشروط الحدودية (25) نجد أن:

$B_{v} \lambda_{v}=0$

$-A_{v} \lambda_{v} \sin \lambda_{v}+B_{v} \lambda_{v} \cos \lambda_{v}=0$

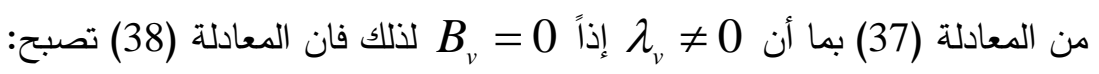
$-A_{v} \lambda_{v} \sin \lambda_{v}=0$

بما أن

$\sin \lambda_{v}=0$

$\Rightarrow \lambda_{v}=n \pi \quad, \quad n=1,2,3, \ldots$

بما أن $F(X)=A_{v} \cos \lambda_{v} X$

$\lambda_{v}=n \pi \quad, \quad n=1,2,3, \ldots$

بما أن الحل (36) هو أحد حدود الحل المفروض (35)، لذلك فـان الحل (35)، ولكي يحقق الثروط الحدودية (25) يجب أن يكون بالثكل الأتي:

$F(X)=\sum_{v=1}^{\infty} A_{v} \cos \lambda_{v} X$ 
$\lambda_{v}=n \pi \quad, \quad n=1,2,3, \ldots$

بتعويض المعادلة (39) في المعادلة (24) نحصل على :

$\sum_{v=1}^{\infty}\left(-\lambda_{v}^{2} A_{v} \cos \lambda_{v} X\right)+\sum_{v=1}^{\infty} A_{v}\left(\frac{r L^{2}}{D}-k^{2}-k c_{2}-\frac{2 r L^{2}}{D} u_{1}(X)\right) \cos \lambda_{v} X=0$

حسب طريقة Galerkin فان التقريب هنا لا يساوي صفراً بل هو نسبة خطأ (Error) والذي يطلق عليه المتبقي (Residual) أي أن : فان

$\sum_{v=1}^{p}\left(-\lambda_{v}^{2} A_{v} \cos \lambda_{v} X\right)+\sum_{v=1}^{p} A_{v}\left(\frac{r L^{2}}{D}-k^{2}-k c_{2}-\frac{2 r L^{2}}{D} u_{1}(X)\right) \cos \lambda_{v} X=\epsilon$

وحيث أن هذا الخطأ أو المتبقي (G) وحسب طريقة Galerkin نسعى إلى جعله اقل ما

يمكن (Minimize the error)، وان التكامل المحدد بالثروط الحدودية للمتبقي السابق مضروباً بدالة يكون مساويا للصفر [1] لذلك فان:

$$
\begin{aligned}
& \int_{0}^{1}\left[\sum_{v=1}^{p} \sum_{m=1}^{p}\left(-\lambda_{v}^{2} A_{v} \cos \lambda_{v} X\right) \varphi_{m}(X)+\right. \\
& \left.+\sum_{v=1}^{p} \sum_{m=1}^{p}\left(\frac{r L^{2}}{D}-k^{2}-k c_{2}-\frac{2 r L^{2}}{D} u_{1}(X)\right)\left(A_{v} \cos \lambda_{v} X\right) \varphi_{m}(X)\right] d X=0
\end{aligned}
$$

حيث أن

$\varphi_{m}(X)=\sin \lambda_{m} X$

$\lambda_{m}=m \pi \quad, \quad m=1,2,3, \ldots$

من المعادلة (40) نحصل على :

$\frac{r L^{2}}{D}-k^{2}-k c_{2}-\frac{2 r L^{2}}{D} u_{1}(X)=\lambda_{v}^{2}$

$\Rightarrow \frac{r L^{2}}{D}-k^{2}-k c_{2}-\frac{2 r L^{2}}{D} u_{1}(X)=(n \pi)^{2}$

$\Rightarrow c_{2}=\frac{-k^{2} D-2 r L^{2} u_{1}(X)-(n \pi)^{2} D+r L^{2}}{k D}$

نلاحظ أن المعادلة (41) هي مطابقة للحل التحليلي (31) وهذا يدل على كفاءة طريقة Galerkin العددية وكذلك يبرهن على صحة الحل التحليلي الذي تم التوصل إليه في إيجاد شروط الاستقرارية لمعادلة Fisher في حالة السعة المتغيرة. 


\section{المصادر}

[1] Allaire, P. E. (1985) Basics of the Finite Element Method, Wm. C. Brown Publishers.

[2] Fisher, R.A. (1937) "The Wave of Advance of Advantageous Genes", Annals of Eugenics 7, pp. 355-369.

[3] Fory's, U. and A. Marciniak-Czochra, (2003) "Logistic Equations in Tumour Growth Modelling", Int. J. Appl. Math. Comput. Sci., Vol. 13, No. 3, pp. 317-325.

[4] Gourley, S.A. (2000) "Travelling Front Solutions of a Nonlocal Fisher Equation”, J. Math. Biol. 41, pp. 272-284.

[5] Logan, J. D. (1987) Applied Mathematics, John Wiley and Sons.

[6] Manaa, Saad A. and B.M. Ibrahim, (2004) "Stability Analysis for A Fully Developed Laminar Fluid Flow in A Rectangular Bend with Secondary Flow", Raf. Jour. Sci., Vol. 15, No.1, Math \& Stati., Special Issue, pp. 146-152.

[7] Ortega-Cejas, V.; J. Fort, and V. Mendez, (2004) "The Role of The Delay Time in The Modeling of Biological Range Expansions", J. Ecology, 85 (1), pp. 258-264.

[8] Pesin, Y. and A. Yurchenko, (2004) "Some Physical Models of The Reaction-Diffusion Equation and Coupled Map Lattices", Russian Math. Surveys, Vol. 8, No. 3,pp.177-218.

[9] Puri, S. and Wiese, K. J. (2003) "Perturbative Linearization of Reaction-Diffusion Equations", J. Phys., A36, pp.2043-2054.

[10] Sasaki, T. (2004) "The Effect of Local Prevention in an Sis Model With Diffusion", Discrete and Continuous Dynamical Systems Series B , Vol. 4, No. 3 , PP. 739-746.

[11] Smith, G. D. (1965) Numerical Solution of Partial Differential Equations, Oxford University Press, 